\title{
A Cyclosymmetric Beam Model and a Spring-Supported Annular Plate Model for Automotive Disc Brake Vibration
}

\author{
Dennis Boennen and Stephen James Walsh \\ Department of Aeronautical and Automotive Engineering, Loughborough University, Leicestershire LE11 3TU, UK \\ Correspondence should be addressed to Stephen James Walsh, s.j.walsh@lboro.ac.uk \\ Received 30 May 2012; Accepted 25 June 2012 \\ Academic Editors: R. Nagaosa and S. Shen
}

Copyright ( $) 2012$ D. Boennen and S. J. Walsh. This is an open access article distributed under the Creative Commons Attribution License, which permits unrestricted use, distribution, and reproduction in any medium, provided the original work is properly cited.

\begin{abstract}
This paper discusses two simplified analytical models for automotive disc brake vibration which can be used to complement more complex finite element methods. The first model approximates the brake disc as a simple beam structure with cyclosymmetric boundary conditions. Since the beam model is a one-dimensional approach, modelling of the inner boundary condition of the brake disc, at the interface of the brake rotor and the central hat, is not possible. The second model, which is established based upon Kirchhoff's thin plate theory, is presented in this paper in order to incorporate the vibrational deformation at the hat-disc interface. The mode shapes, natural frequencies, and forced response of a static disc are calculated using different inner boundary conditions. Among others, the spring-supported boundary condition is proposed and applied in this paper to make appropriate predictions. The predicted results are compared with measurements of the vibration characteristics of a solid brake disc mounted upon a static test rig. These comparisons demonstrate that the most appropriate model for the inner boundary condition of the measured brake disc is the proposed spring-supported inner boundary condition.
\end{abstract}

\section{Introduction}

The investigation of brake squeal is of continuing interest to the automotive industry due to the high warranty costs that have to be paid every year to replace complete or individual parts of the brake systems. References [1-6] present a useful review of current knowledge within the public domain. Chen [1] noted that brake squeal is generated by a dynamic instability within the brake system. The mechanisms of this instability can be broadly categorised into two groups: (i) friction force-induced excitation and (ii) friction force-induced structural coupling. Modelling of brake disc vibration is one of the key factors in understanding and controlling squeal generation, as the squeal frequencies are almost always at or near to the resonant frequencies of the disc [1].

Finite element models are widely used to investigate brake system instability and have given considerable insight to the problem. Specific examples of how the geometric complexity of a braking system can be modelled using finite elements is discussed by Trichês Jr et al. [7] and Dai and
Lim [8]. To complement the use of finite element models various lumped parameter or continuous system analytical models have been developed. For example, in modelling the brake disc, Flint and Hultén $[9,10]$ have used a onedimensional beam-bending model whilst Ouyang and his colleagues [11-13] have applied a flat plate bending model. Previous research has noted that the in-plane vibrations of a disc can also contribute to brake squeal generation [1]. However, Cao et al. [14] reported that the in-plane motion was found to have a low influence in the frequency range below $6 \mathrm{kHz}$. Chen et al. and Bae and Wickert $[2,15]$ present a good illustration of the variation in the natural frequencies of bending and in-plane modes of a solid annular disc as a function of thickness to diameter ratio. For a relatively thick disc the lowest in-plane mode may fall in the same frequency range as the lowest bending mode. A detailed theoretical description of the in-plane vibration of circular annular discs is presented by Bashmal et al. [16].

In this paper two analytical models for automotive disc brake vibration are presented. Of course, simplified analytical models will not be able to represent the complexity 
of mechanisms involved in practical brake system vibration. However, as noted by Papinniemi and his coauthors [5] they can provide insight by highlighting the physical phenomena that occur. They can also assist in the interpretation of experimental results and the development of improved computational tools. The first model presented in this paper approximates the brake disc as a simple beam structure with cyclosymmetric boundary conditions. This model follows the approach presented by Flint and Hultén [9, 10]. The aim of this investigation is to assess whether any further guidance can be provided on the appropriate frequency range of applicability of the cyclosymmetric beam model. Predicted results from a travelling wave model are compared to predictions of the forced response from a separately derived modal-based model of the structure. Predicted results are also compared to experimental measurements made upon a large diameter ring, a solid brake disc with its central hat removed, and a standard production nonvented brake disc.

Since the beam model is a one-dimensional approach and, therefore, the modelling of the inner boundary conditions of the brake disc is impossible, a second model based upon Kirchhoff's thin plate theory is also presented. The mode shapes and natural frequencies of a static disc with clamped, simply-supported, and free inner boundary conditions are calculated. A novel approach of simulating the inner boundary by using an infinite number of springs with a spring stiffness calculated using the geometry of the central hat is also developed. The purpose of this investigation is to discuss the appropriateness of an alternative boundary condition in comparison to the commonly applied clamped inner boundary condition. It is hoped that this boundary condition may approximate some of the deformation at the hat-disc interface while avoiding the complexity of a fully coupled hat-disc analytical model. Predicted natural frequencies are compared to experimentally measured resonant frequencies of a brake disc rigidly mounted upon a static test rig. Using the spring-supported inner boundary condition a forced vibration model is developed and compared to the experimentally measured forced response of the structure. As noted above, in-plane vibrations become more significant at higher frequencies and for relatively thick discs. In this paper only the out-of-plane vibrations of the disc are modelled, and, thus, the investigation is relevant to the lower-frequency modes of a brake disc.

\section{Beam Model}

2.1. Wave-Based Approach. In this approach the brake disc is modelled as a ring, which is assumed to be unwrapped into a straight Euler-Bernoulli beam with cyclosymmetric boundary conditions at each end. The derivation below follows the approach of Flint and Hultén [9, 10] who modelled the disc as an Euler-Bernoulli beam with and without an elastic foundation. However, unlike $[9,10]$, in this paper the boundary conditions at the ends of the beam are solved explicitly to obtain the amplitudes of the constituent waves in the vibration. Further details of this approach are given in $[17,18]$.
Consider a straight beam of finite length $2 L$ excited by a force at the point $x=0$ as shown in Figure 1. $A_{1}$ and $A_{2}$ represent the amplitudes of the near field waves close to the force, and $A_{3}$ and $A_{4}$ represent the amplitudes of the propagating waves, which travel away from the forced excitation. Cyclosymmetric boundary conditions at both ends imply that the coefficients $A_{8}, A_{7}, A_{6}$, and $A_{5}$ represent the amplitudes $A_{1}, A_{2}, A_{3}$, and $A_{4}$, respectively, continuing beyond the boundaries. The equation of motion of the system is given by

$$
\begin{aligned}
& \frac{\partial^{2}}{\partial x^{2}} \cdot\left[E \cdot I(x) \cdot\left(\frac{\partial^{2} u(x, t)}{\partial x^{2}}\right)\right]+\rho \cdot A(x) \cdot \frac{\partial^{2} u(x, t)}{\partial t^{2}} \\
& \quad=f(x, t),
\end{aligned}
$$

where $E$ is Young's modulus, $I$ is the moment of inertia, $\rho$ is the density, $A(x)$ is the area of cross-section of the beam, $u(x)$ is the displacement, and $f(x, t)$ is the applied force. The solution of the matrix system of equations for the boundary conditions yields the following wave amplitude relationships:

$$
\begin{gathered}
A_{1}=A_{2}=-\frac{F_{0} \cdot e^{k \cdot L}}{4 \cdot E \cdot I \cdot k^{3} \cdot\left(e^{k \cdot L}-e^{-k \cdot L}\right)}, \\
A_{3}=A_{4}=-j \cdot \frac{F_{0}}{4 \cdot E \cdot I \cdot k^{3}} \cdot \frac{e^{j \cdot k \cdot L}}{\left(e^{j \cdot k \cdot L}-e^{-j \cdot k \cdot L}\right)}, \\
A_{5}=A_{6}=-j \cdot \frac{F_{0}}{4 \cdot E \cdot I \cdot k^{3}} \cdot \frac{e^{-j \cdot k \cdot L}}{\left(e^{j \cdot k \cdot L}-e^{-j \cdot k \cdot L}\right)}, \\
A_{7}=A_{8}=-\frac{F_{0} \cdot e^{-k \cdot L}}{4 \cdot E \cdot I \cdot k^{3} \cdot\left(e^{k \cdot L}-e^{-k \cdot L}\right)},
\end{gathered}
$$

where $F_{0}$ is the amplitude of the force, $k$ is the wavenumber, and $L$ is half of the length of the beam. As expected, these amplitude coefficients show the symmetry of wave motion around the excitation location, $x=0$. Thus, a prediction of the theoretical value of the point receptance at the excitation location can be obtained by assuming unit force, $F_{0}=1.0$, and summing the derived wave amplitudes, $A_{1}-A_{8}$ to form the total displacement, $u(0)$.

2.2. Modal-Based Approach. In an alternative to the wavebased approach, the cyclosymmetric beam was also modelled using a modal summation approach [19]. Thus, the velocity response, $v(x)$, to any arbitrary excitation in terms of the eigenfunctions and eigenfrequencies of the system, is given by

$$
\begin{aligned}
v(x)= & j \cdot \omega \cdot \sum_{n=0}^{\infty} \frac{\varphi_{n}(x)}{\Lambda_{n} \cdot\left(\omega_{n}^{2} \cdot(1+j \cdot \eta)-\omega^{2}\right)} \\
& \cdot \int p(x) \cdot \varphi(x) \cdot d x,
\end{aligned}
$$

where $\eta$ is the loss factor, $p(x)$ is defined as the exciting pressure, and the norm or modal mass $\Lambda$ is defined as

$$
\Lambda_{n}=\int_{-L}^{L} \rho \cdot A \cdot \varphi_{n}(x)^{2} d x .
$$




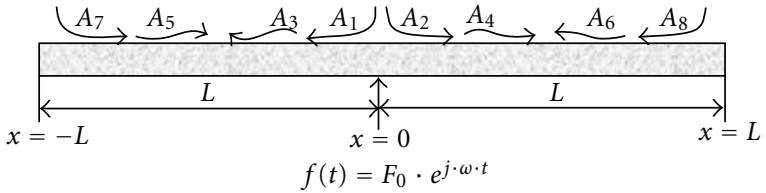

Figure 1: A cyclosymmetric beam of length $2 L$ excited by a point force, $f(t)$, at the origin, $x=0$.

For the case of a point force excitation at the centre of the beam the eigenfunctions are defined as

$$
\varphi_{n}(x)=\cos (k \cdot L) .
$$

For the case of free vibration additional eigenfunctions, $\phi_{n}(x)=\sin (k \cdot L)$ should also be included. Using the cyclosymmetric boundary conditions a matrix system of equations can be constructed and setting the determinant of this matrix system equal to zero gives the eigenfrequencies of this system as

$$
\omega_{n}=\frac{n \cdot \pi}{L}
$$

At the point of excitation $x^{\prime}$ the force integral can be reduced to

$$
\int p(x) \cdot \phi(x) \cdot d x=F_{0} \cdot \phi\left(x^{\prime}\right) .
$$

Thus, using (3) a prediction of the theoretical value of the point mobility at the excitation location can be obtained by assuming unit force, $F_{0}=1.0$. In Figure 2 the modulus of the point mobility of the cyclosymmetric beam predicted by the modal summation approach is compared to a prediction made using the wave-based approach. For the modal summation approach the modes from $n=0$ to $n=15$ are included. The geometrical values and material properties used are those of the large diameter experimental ring described in Section 3. It can be seen in Figure 2 that both the wave-based approach of Flint and Hulten $[9,10]$ and the modal summation approach predict identical results.

\section{Annular Plate Model}

The scope of this section is to develop free and forced vibration models for a solid, nonvented, and brake disc by using annular plate theory. Four different cases for the inner boundary are considered: (i) free, (ii) simply supported; (iii) clamped, and (iv) spring supported. In each case the outer boundary is assumed to be free. The standard boundary conditions for a brake disc model assume that the displacement at the inner boundary is zero. This assumption has been applied successfully by many researchers $[1,2]$. However, this assumption may not be true for all designs of brake disk. In [4] an example was reported where the undercut of the rotor at the hat-disc interface was deepened in order to help reduce brake disc squeal. A design change such as this may well violate the clamped inner boundary assumption.

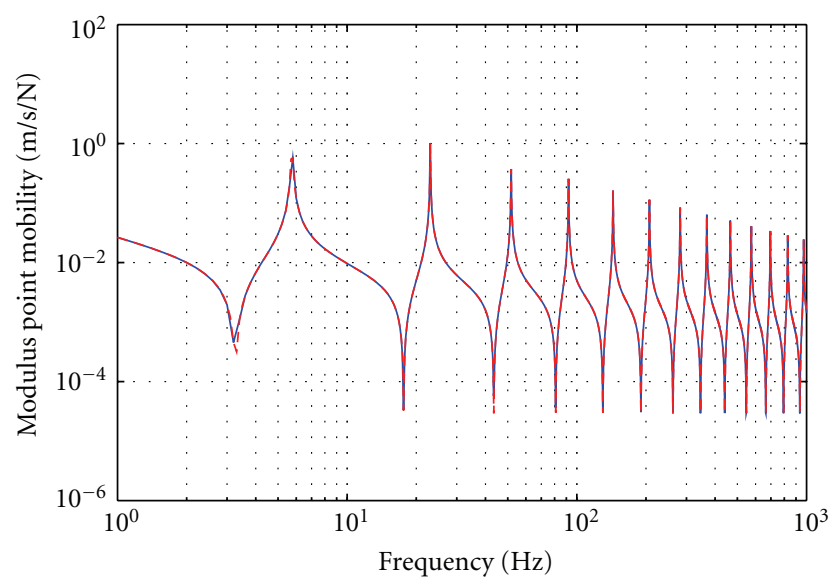

Figure 2: Predicted modulus of the point mobility of the large diameter experimental ring modelled as a cyclosymmetric beam with $L=1428 \mathrm{~mm}$ using the wave based approach (solid line) and the modal based approach (dashed line).

In the following section a brief summary of annular plate theory relevant to a disc with different boundary conditions is given. This is followed by a presentation of the existing models of disc vibrations used by various authors [20-22] to model boundary conditions (i) -(iii) above. To extend this analysis, a novel approach based on case (iv), a spring-supported inner boundary, is developed. Finally, in Section 3.2, equations for the forced response of the structure are developed by incorporating the spring-supported inner boundary condition.

3.1. Free Vibration Model. The theory used, following Kirchhoff, assumes small deflections and neglects the influence of rotary inertia and the additional deflections caused by shear deformation. In Figure 3 a sketch of a free-free disc is shown, where $a$ and $b$ are the inner and outer radii distances, respectively, from the centre of the disc, $r=0$, and the thickness is stated as $h$. The differential equation of motion governing the displacement $u(r, \theta, t)$ of a point along the central plane of the plate can be written in polar coordinates, $(r, \theta)$, in the form

$$
D \cdot \nabla^{4} \cdot u(r, \theta, t)+\rho \cdot h \cdot \frac{\partial^{2} u(r, \theta, t)}{\partial t^{2}}=0,
$$

where the flexural rigidity $D$ and the Laplacian operator $\nabla^{2}$ are defined as

$$
\begin{gathered}
D=\frac{E \cdot h^{3}}{12 \cdot\left(1-\nu^{2}\right)}, \\
\nabla^{2}=\frac{\partial^{2}}{\partial r^{2}}+\frac{1}{r} \cdot \frac{\partial}{\partial r}+\frac{1}{r^{2}} \cdot \frac{\partial^{2}}{\partial \theta^{2}},
\end{gathered}
$$

where $v$ is Poisson's ratio and $\nabla^{4}$ is defined as

$$
\nabla^{4}=\nabla^{2} \cdot \nabla^{2} \text {. }
$$

A solution of the following type for a typical mode can be assumed as

$$
u(r, \theta, t)=U(r, \theta) \cdot \sin \left(\omega \cdot\left(t-t_{0}\right)\right) .
$$


The general solution can be written in polar coordinates as

$$
\begin{aligned}
U(r, \theta)=\sum_{n=0}^{\infty}\left[A \cdot J_{n}(k r)+B \cdot Y_{n}(k r)+C \cdot I_{n}(k r)\right. \\
\left.\quad+D^{*} \cdot K_{n}(k r)\right] \cdot \cos (n \cdot \theta)+ \\
\sum_{n=1}^{\infty}\left[A^{*} \cdot J_{n}(k r)+B^{*} \cdot Y_{n}(k r)+C^{*} \cdot I_{n}(k r)\right. \\
\left.\quad+D^{* *} \cdot K_{n}(k r)\right] \cdot \sin (n \cdot \theta),
\end{aligned}
$$

where the wavenumber, $k$, is given by

$$
k^{4}=\frac{\rho \cdot \omega^{2}}{D},
$$

where $J_{n}$ is a Bessel function of the first order, $Y_{n}$ is a Bessel function of the second order, and $I_{n}$ and $K_{n}$ are modified Bessel functions of the first and second order, respectively. The unknown wave amplitudes of the Bessel functions are given by $A, A^{*}, B, B^{*}, C, C^{*}, D^{*}$, and $D^{* *}$. For this analysis, the terms involving $\sin (n \theta)$ are not taken into consideration, since the boundary conditions possess symmetry with respect to one or more diameters of the annular plate. Therefore, the following displacement expression can be made for a typical mode of the disc as follows:

$$
\begin{aligned}
U(r, \theta)=( & A \cdot J_{n}(k r)+B \cdot Y_{n}(k r) \\
& \left.+C \cdot I_{n}(k r)+D^{*} \cdot K_{n}(k r)\right) \cdot \cos (n \cdot \theta) .
\end{aligned}
$$

Expressions for the shear force, the bending moment, and the twisting moment are needed to define the boundary conditions. The bending moment in radial direction can be expressed as

$$
\begin{aligned}
M_{r}(r, \theta, t)=D \cdot( & \frac{\partial^{2} u(r, \theta, t)}{\partial r^{2}} \\
& \left.+v \cdot\left(\frac{1}{r} \cdot \frac{\partial u(r, \theta, t)}{\partial r}+\frac{1}{r^{2}} \cdot \frac{\partial^{2} u(r, \theta, t)}{\partial \theta^{2}}\right)\right),
\end{aligned}
$$

where the shear force in radial direction is defined as

$$
Q_{r}(r, \theta, t)=-D \cdot \frac{\partial}{\partial r} \cdot \nabla^{2} u(r, \theta, t),
$$

and the twisting moment is defined as

$$
M_{r \theta}(r, \theta, t)=-D \cdot(1-v) \cdot \frac{\partial}{\partial r} \cdot\left(\frac{1}{r} \cdot \frac{\partial u(r, \theta, t)}{\partial \theta}\right) .
$$

Defining the boundary condition at a free edge is more complex, because at a free edge the shear force, the bending moment, and the twisting moment must all be equal to zero. In order to avoid an overdetermined systems of equations

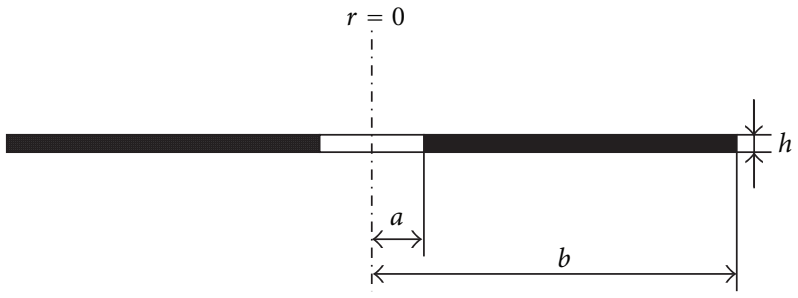

FIGURE 3: Sketch of the free-free disc, sectional view.

only two boundary conditions for each edge are required. Therefore, the Kelvin-Kirchhoff edge reaction has been defined, which incorporates both the shear force in the radial direction and the twisting moment. Thus,

$$
V_{r}(r, \theta, t)=Q_{r}(r, \theta, t)+\frac{1}{r} \cdot \frac{\partial M_{r \theta}(r, \theta, t)}{\partial \theta} .
$$

The simply supported and clamped inner boundary conditions assumed by previous authors [20-22] are listed in Table 1. For completeness, the free-free inner and outer boundary conditions of a ring are also shown.

In the following approach a novel inner boundary is developed whereby the hat is modelled as springs with an equivalent stiffness relating the geometry of the hat and its Young's modulus. At the inner boundary of the disc a spring force is assumed to act as a distributed force per unit length along a tiny element of the circumference of length $d U$, and, hence, to be equal to the Kelvin-Kirchhoff edge condition $V_{r}(r, \theta, t)$, acting along the same tiny element of the circumference $d U$ as shown in Figure 4. Since the KelvinKirchoff edge condition, $V_{r}(r, \theta, t)$, is defined as force per unit length parallel to the circumferential direction $\theta$ [23], then

$$
f_{s}(r, \theta, t) \cdot d U=V_{r}(r, \theta, t) \cdot d U,
$$

with the spring force per unit length, $f_{s}(r, \theta, t)$, given by

$$
f_{s}(r, \theta, t)=k_{s} \cdot u(r, \theta, t) .
$$

By applying Hook's law to a tiny element of length $d U$ along the circumference of the cylindrical wall of the central hat as shown in Figure 4 the following equation can be derived:

$$
u(r, \theta, t)=\frac{f_{s}(r, \theta, t) \cdot d U \cdot L_{0}}{E \cdot A_{e}}
$$

where $L_{0}$ is the height of the hat of the disc. The crosssectional area, $A_{e}$, of a tiny element of the cylindrical wall of the hat can be approximated by the rectangular dimensions

$$
A_{e}=d U \cdot\left(r_{i 2}-r_{i 1}\right),
$$

where $r_{i 2}$ is defined as the outer radius of the hat and $r_{i 1}$ as the inner radius. By using (20)-(22) an expression for the equivalent spring stiffness per unit length, $k_{s}$, can be found as

$$
k_{s}=\frac{E \cdot\left(r_{i 2}-r_{i 1}\right)}{L_{0}} .
$$


TABLE 1: Boundary conditions for the brake disc model.

\begin{tabular}{lccc}
\hline & Free-free & Simply supported free & Clamped free \\
\hline Inner boundary, $r=a$ & $M_{r}(a, \theta, t)=0$ & $u(a, \theta, t)=0$ & $u(a, \theta, t)=0$ \\
& $V_{r}(a, \theta, t)=0$ & $M_{r}(a, \theta, t)=0$ & $\partial u(a, \theta, t) / \partial r=0$ \\
Outer boundary, $r=b$ & & $M_{r}(b, \theta, t)=0$ & $V_{r}(b, \theta, t)=0$ \\
\hline
\end{tabular}

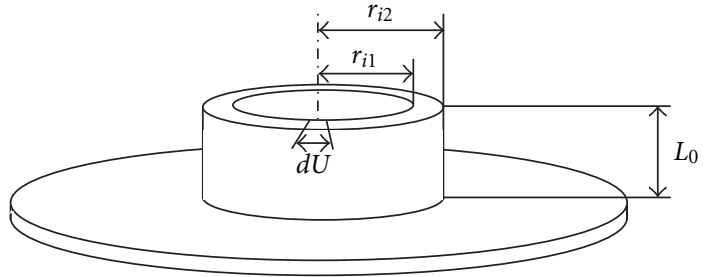

FIGURE 4: Sketch of the brake disc including the hat dimensions: inner radius, $r_{i 1}$; outer radius, $r_{i 2}$; height, $L_{0}$; element length, $d U$.

Substituting (23) into (19) leads to the following boundary condition at the inner edge of the disc, $r=a$ :

$$
V_{r}(a, \theta, t)-u(a, \theta, t) \cdot \frac{E \cdot\left(r_{i 2}-r_{i 1}\right)}{L_{0}}=0 .
$$

Thus, for the first inner boundary condition of the disc, the Kelvin-Kirchoff edge condition is related to the displacement, the geometry of the hat, and its Young's modulus. Note that the inner radius of the disc, $r=a$, is at the same distance as the inner radius of the hat, $r=r_{i 1}$. For the second inner boundary condition the bending moment in radial direction, $M_{r}(a, \theta, t)$, is assumed to be zero at $r=a$.

Figure 5 shows the effect of the length of the hat, $L_{0}$, on the predicted natural frequencies of a disc modelled with a spring-supported inner boundary condition. The natural frequencies are plotted for the second, third, fourth, and fifth diametral modes. The disc is assumed to have the same material properties as the standard production nonvented brake disc listed in Table 2. Similarly the inner and outer hat radii and the disc thickness are the same as the standard production nonvented brake disc. It can be seen in Figure 5 that as the hat length decreases, the predicted natural frequency for each mode increases. This is to be expected as (23) indicates that as the length $L_{0}$ decreases the stiffness, $k_{s}$, increases. Hence, the spring supported boundary condition approaches that of the simply supported boundary condition. Conversely, as the hat length increases the predicted natural frequency for each mode decreases. This also is to be expected as (23) indicates that as the length $L_{0}$ increases the stiffness, $k_{s}$, decreases. Hence, the spring supported boundary condition approaches that of the free edge, where the Kelvin-Kirchoff edge condition, $V_{r}$, is zero. The hat length, $L_{0}$, marked with a vertical line is the actual length of the hat of standard production nonvented brake disc. At this typical hat length the predicted natural frequencies of the spring supported model lie closer to that of the simply supported boundary condition given by the extremely short lengths at the left hand side of Figure 5

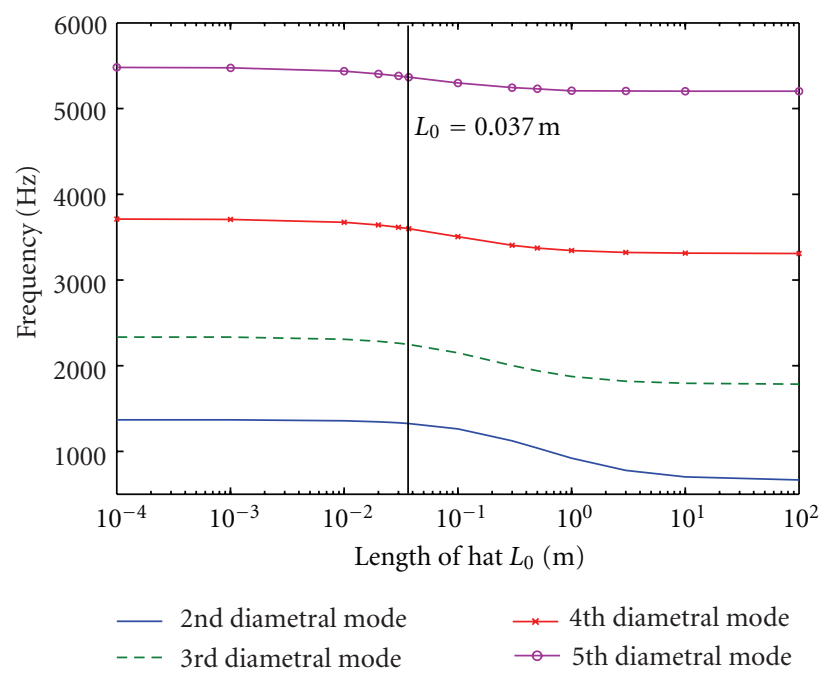

FIGURE 5: Effect of the length of hat, $L_{0}$, on the predicted natural frequencies of the $2 \mathrm{nd}, 3 \mathrm{rd}, 4 \mathrm{th}$, and 5 th diametral mode of a disc modelled with a spring supported inner boundary condition.

rather than the free boundary condition represented by the extremely long lengths at the right had side of the Figure 5.

3.2. Forced Vibration Model. For the forced vibration model the modal summation approach of Section 2.2 is extended to the case of a disc with a spring-supported inner boundary. The response velocity of the disc at location $(r, \theta)$ is given by

$$
\begin{aligned}
v\left(r, \theta, r^{\prime}, \theta^{\prime}\right)= & j \cdot \omega \cdot \sum_{c=1}^{\infty} \sum_{n=1}^{\infty} \frac{R_{c n}(r) \cdot \cos \left(n \cdot\left(\theta-\theta^{\prime}\right)\right)}{\Lambda_{c n} \cdot\left(\omega_{c n}^{2} \cdot(1+j \cdot \eta)-\omega^{2}\right)} \\
& \cdot \int p\left(r^{\prime}, \theta^{\prime}\right) \cdot R_{c n}\left(r^{\prime}\right) \cdot r^{\prime} \cdot d r
\end{aligned}
$$

where $R_{c n}$ is a linear combination of Bessel functions. The modal mass or norm $\Lambda$ is defined as

$$
\Lambda_{c n}=\int_{a}^{b} \int_{0}^{2 \cdot \pi} \rho \cdot h \cdot\left(R_{c n}(r) \cdot \cos \left(n \cdot\left(\theta-\theta^{\prime}\right)\right)\right)^{2} \cdot r \cdot d r \cdot d \theta .
$$

The force integral at the point of excitation, $\left(r^{\prime}, \theta^{\prime}\right)$, can be simplified to

$$
\begin{aligned}
& \int p\left(r^{\prime}, \theta^{\prime}\right) \cdot R_{c n}\left(r^{\prime}\right) \cdot \cos \left(n \cdot\left(\theta-\theta^{\prime}\right)\right) \cdot r \cdot d r \cdot d \theta \\
& =R_{c n}\left(r^{\prime}\right) \cdot F_{0} .
\end{aligned}
$$


TABLE 2: Dimensions and material properties of the experimental test structures.

\begin{tabular}{lccccc}
\hline Test structure & $\begin{array}{c}\text { Young's } \\
\text { modulus, } \\
E \mathrm{~N} / \mathrm{m}^{2}\end{array}$ & $\begin{array}{c}\text { Density, } \\
\rho \mathrm{kg} / \mathrm{m}^{3}\end{array}$ & $\begin{array}{c}\text { Poisson's } \\
\text { ratio, } v\end{array}$ & $\begin{array}{c}\text { Outer } \\
\text { diameter, } \\
\emptyset_{\text {outside }} \mathrm{m}\end{array}$ & $\begin{array}{c}\text { Inner diameter, } \\
\emptyset_{\text {inside }} \mathrm{m}\end{array}$ \\
\hline $\begin{array}{l}\text { Large diameter ring } \\
\begin{array}{l}\text { Small diameter } \\
\text { annular plate }\end{array}\end{array}$ & $\begin{array}{c}2.1 \times 10^{11} \\
1.2 \times 10^{11}\end{array}$ & 7850 & 0.3 & $950 \times 10^{-3}$ & $850 \times 10^{-3}$ \\
$\begin{array}{l}\text { Standard production } \\
\text { nonvented brake disc }\end{array}$ & $1.2 \times 10^{11}$ & 7250 & 0.26 & $262.3 \times 10^{-3}$ & $160 \times 10^{-3}$ \\
\hline
\end{tabular}

The spring-supported inner boundary condition at $r=a$ derived in the Section 3.1 can now be used to predict the vibration response of the structure. The time-independent part, $R_{c n}$, is defined by (28), where the subscript $c$ stands for the circular and the subscript $n$ for the diametral modes as follows:

$$
\begin{aligned}
R_{c n}(r)=\left(J_{n}\left(k_{c n} r\right)+\frac{B}{A} \cdot Y_{n}\left(k_{c n} r\right)\right. \\
\left.\quad+\frac{C}{A} \cdot I_{n}\left(k_{c n} r\right)+\frac{D^{*}}{A} \cdot K_{n}\left(k_{c n} r\right)\right) .
\end{aligned}
$$

\section{Experimental Apparatus and Measurement Method}

To verify the predictions made from the cyclosymmetric beam model described in Section 2 measurements were made of the vibrational behaviour of a number of test structures: (i) a large diameter ring, (ii) a small diameter annular plate, and (iii) a standard production nonvented brake disc. The dimensions and material properties of the test structures are listed in Table 2.

The large diameter steel ring was selected because of its mild curvature and small ring width of $50 \mathrm{~mm}$. Hence, this structure would be expected to exhibit the vibrational characteristics of a straight beam with cyclosymmetric boundary conditions over a wide frequency range. To measure the frequency response function the ring was suspended vertically on thin wires from a large frame and excited by an electrodynamic exciter using a random noise input over the frequency range from 0 to $3200 \mathrm{~Hz}$. The point response of the ring was measured by using a force transducer and a lightweight accelerometer at excitation location. To identify the relevant mode shapes accelerometer measurements were made at sixteen equidistant locations around the centre line of the ring midradius distance between the inner and outer edges.

The small diameter annular plate was obtained by taking a nonvented brake disc and removing its central hat, thus, forming an annular plate with the dimensions and material properties similar to that of a standard production brake disc. The frequency response functions and mode shapes were measured in the same manner as for the large diameter ring.
In order to compare the simply supported-free, clampedfree and spring supported-free annular plate models, developed in Section 3 with experimental measurements a standard production brake disc was attached with fixing bolts onto a wheel rigidly mounted onto a large and heavy steel frame. Applied force and response acceleration measurements were taken in the same manner as before. In order to distinguish between the different circumferential modes of the disc, response acceleration measurements were taken in the radial as well as the circumferential directions.

\section{Results and Discussion}

5.1. Large Diameter Steel Ring versus Cyclosymmetric Beam Model. Figure 6 shows a comparison of the modulus of the measured point mobility of the experimental steel ring (dashed line) with a theoretical prediction of the point mobility from the cyclosymmetric beam model developed in Section 2 (solid line) over a frequency range from 1 to $3200 \mathrm{~Hz}$. The cyclosymmetric beam model was assumed to have a half-length, $L$, of $1428 \mathrm{~mm}$. Also shown in Figure 6 is the modulus of the point mobility, $Y(\omega)$, of the equivalent "infinite" beam, given in [19] as

$$
Y(\omega)=\frac{(1-j)}{4 \cdot A \cdot \rho \cdot \sqrt{\omega}} \cdot\left(\frac{A \cdot \rho}{E \cdot I}\right)^{1 / 4} .
$$

It can be seen in Figure 6 that both the theoretical prediction (solid line) and the measured data (dashed line) follow the trend of the equivalent "infinite" structure, thus, indicating beam-like behaviour. It can also be seen in Figure 6 that above $100 \mathrm{~Hz}$ the predicted natural frequencies of the structure correspond approximately to the measured resonant frequencies of the experimental ring. Figure 7 shows the percentage difference between the measured resonant frequencies and the predicted natural frequencies. Above $100 \mathrm{~Hz}$ the difference is relatively small. Above the frequency of $500 \mathrm{~Hz}$ it is approximately constant $5 \%$. This may be due to incorrect values of the material properties used in the beam model as their values were obtained from standard texts.

At low frequencies it is likely that the effect of curvature will become more significant on the measured data. Previous work on curved beams has shown that curvature effects are important for predominantly flexural waves below approximately $10 \%$ of the ring frequency [24]. For the experimental ring this value is $183 \mathrm{~Hz}$. Although for the experimental steel 


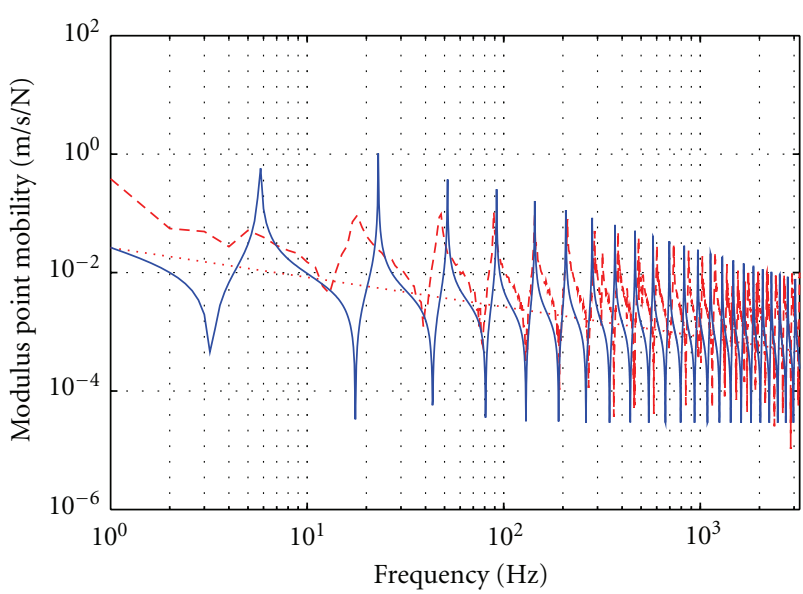

Figure 6: Modulus of the point mobility of the large diameter experimental steel ring: (solid line) theoretical prediction from the cyclosymmetric beam model; (dashed line) measured results; and (dotted line) equivalent "infinite" beam.

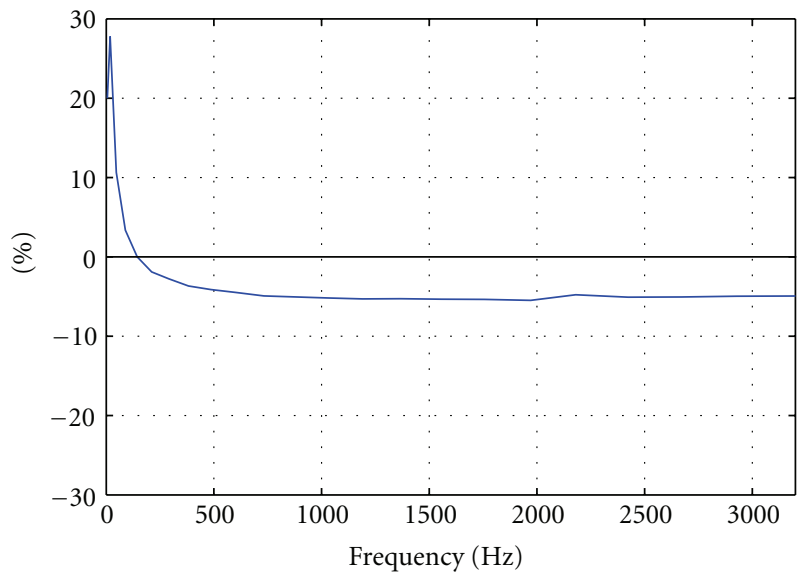

FIGURE 7: Difference in percentage between the measured resonant frequencies of the large diameter steel ring and the predicted natural frequencies of the cyclosymmetric beam model.

ring considered here the bending waves act in a different direction to the curved beam considered in [24], this value can act as a useful guide to the lower frequency limit of the cyclosymmetric beam model. A calculation of the frequency corresponding to half a bending wavelength across the beam width (outer radius minus inner radius) indicates that circumferential-mode behaviour can be expected above $4690 \mathrm{~Hz}$. This is above the frequency range of interest shown in Figure 6.

\subsection{Small Diameter Annular Plate versus Cyclosymmetric}

Beam Model. Figure 8 shows the measured modulus of the point mobility of the small diameter annular plate (dashed line) compared to the calculated modulus of the point mobility predicted using the beam model with cyclosymmetric boundary conditions (solid line) over the frequency range from 100 to $12600 \mathrm{~Hz}$. It can be seen in Figure 8 that both the theoretical prediction and the measured data

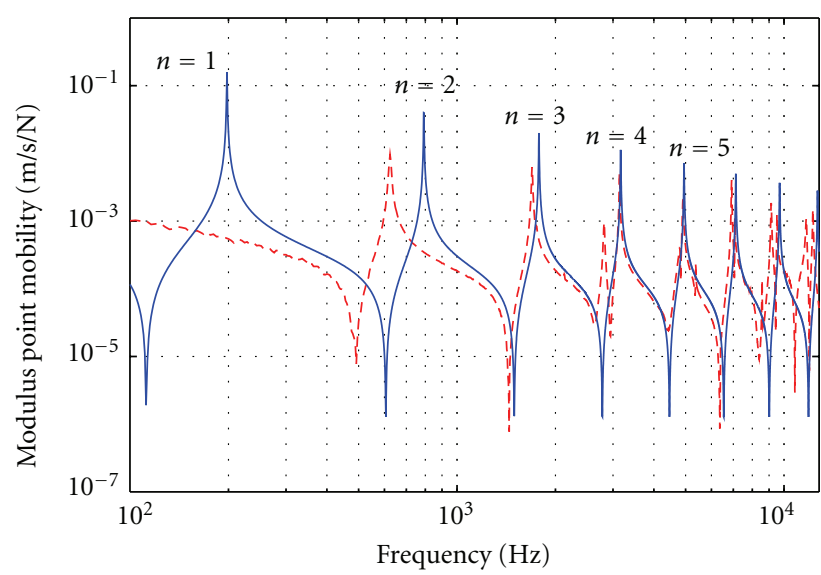

FIgURE 8: Modulus of the point mobility of small diameter annular plate: (solid line) theoretical prediction from the cyclosymmetric beam model with $L=361 \mathrm{~mm}$; (dashed line) measured results.

follow the $1 / \omega^{1 / 2}$ trend with increasing frequency of the equivalent "infinite" beam structure. A comparison between the measured resonant frequencies and calculated natural frequencies predicted by the cyclosymmetric beam model for the $n=2$ to $n=5$ diametral modes is shown in Table 3. It can be seen in Table 3 that apart from the $n=2$ diametral mode the natural frequencies predicted by the cyclosymmetric beam model are similar to the measured resonant frequencies. The discrepancy for the $n=2$ mode is not surprising as the measured resonant frequency at $624 \mathrm{~Hz}$ lies close to the $10 \%$ ring frequency value of $613 \mathrm{~Hz}$ for this structure. Thus, curvature effects may be significant for the $n=2$ diametral mode. However, curvature effects are not included in the cyclosymmetric beam model. Flint and Hultén [9] record a significant difference between the measured resonant frequency and predicted natural frequency for the $n=2$ mode, whilst other diametral modes have relatively good agreement. Thompson [25] has applied a cyclosymmetric beam model to railway wheel bending vibration and compared the predicted natural frequencies to a finite element model of the structure. It is noted in [25] that the $n=0$ and $n=1$ modes are not well represented by the cyclosymmetric beam model. However, the natural frequencies of modes with $1<n<7$ are within $15 \%$ of the corresponding finite element values. For modes with $n>6$, then shear deformation is important, and a Timoshenko beam formulation should be used.

Also apparent in Figure 8 is a smaller amplitude measured resonance at $2800 \mathrm{~Hz}$. This is likely to be the $(1,1)$ circumferential mode predicted by the free-free annular plate model at $2783 \mathrm{~Hz}$. It can be seen in Figure 8 that all of the modes are well separated, and, thus, the circumferential $(1,1)$ mode appears to have little influence upon the adjacent $n=3$ and $n=4$ diametral modes. Circumferential modes are not included in the cyclosymmetric beam model.

The natural frequencies predicted by the free-free annular plate model are also shown in Table 3. The close agreement between the natural frequencies of the free-free annular plate model and the measured resonant frequencies 
TABLE 3: Comparison of the predicted natural frequencies with the measured resonant frequencies of the small diameter annular plate.

\begin{tabular}{lccc}
\hline Diametral mode & Cyclo-symmetric beam model, $f_{\text {calc. }}$ & Free-free plate model, $f_{\text {calc. }}$ & Measured, $f_{\text {meas. }}$ \\
$n$ & $\mathrm{~Hz}$ & $\mathrm{~Hz}$ & $\mathrm{~Hz}$ \\
\hline 2 & 780 & 620 & 1700 \\
3 & 1750 & 3200 & 3140 \\
4 & 3200 & 5000 & 4912 \\
5
\end{tabular}

is a result of the estimation of Young's modulus for the structure. The unknown material properties of a brake disc, which is made out of cast iron, were estimated by correlating the measurement resonant frequencies to the analytical results of the free-free plate model. For this correlation, the Poisson's ratio was kept constant at 0.26 , which is representative for cast iron, and the density was calculated by weighing the structure and measuring its volume giving a result of $7250 \mathrm{~kg} / \mathrm{m}^{3}$. The Young's modulus in the theoretical model was then adjusted until an apparent "best fit" was obtained between the predicted and measured values. The best estimate of the Young's modulus was found to be $1.2 \times$ $10^{11} \mathrm{~N} / \mathrm{m}^{2}$.

5.3. Standard Production Brake Disc versus Cyclosymmetric Beam Model and Annular Plate Models. Figure 9 shows the measured modulus of the point mobility of the rigidly fixed brake disc (solid line) as well as the trend line for the equivalent "infinite" plate calculated from [19] as

$$
Y(\omega)=\frac{\sqrt{12}}{8 \cdot h^{2} \cdot \sqrt{\rho} \cdot \sqrt{E /\left(1-\nu^{2}\right)}} .
$$

It can be seen in Figure 9 that the measured point mobility of the brake disc follows the trend line of the equivalent "infinite" plate, thus, indicating plate-like vibrational behaviour of the disc when its central hat is retained.

The main resonant frequencies and associated diametral modes of the brake disc are labelled in Figure 9 and listed in Table 4. The corresponding mode shapes were identified from the accelerometer measurements around the disc circumference and across its width. In Table 4 the natural frequencies of a simply supported-free, a clampedfree and a spring-supported-free disc calculated using the theoretical models developed in Section 3.1 are compared to the measured resonant frequencies of the brake disc. A comparison between the results listed in Tables 3 and 4 shows that there are considerable differences between the resonant frequencies of the small diameter experimental ring, listed in Table 3, and the resonant frequencies of the standard production nonvented brake disc listed in Table 4. Coupled with the difference in the trend of the point mobility data shown in Figures 8 and 9, this suggests that the inclusion of a central hat in the structure makes its vibrational behaviour platelike in nature.

Comparing the predictions of the annular plate models, it can be seen in Table 4 that there is a discrepancy between the predicted clamped-free natural frequencies and the measured resonant frequencies of the disc. The differences

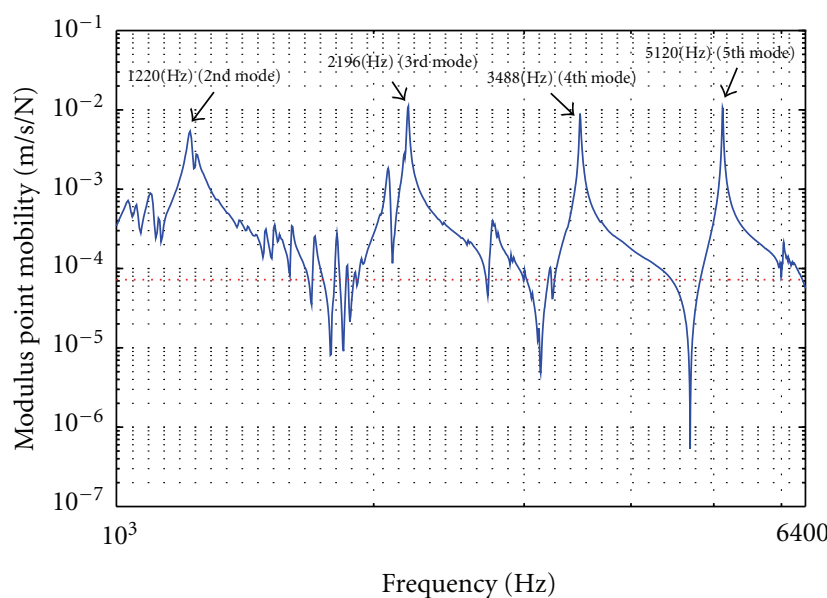

FIgure 9: Modulus of the point mobility of the brake disc over the frequency range 1000 to $6400 \mathrm{~Hz}$, logarithmic scale: (solid line) measured result; (dotted line) equivalent "infinite" beam.

between the simply supported-free model and the measured resonant frequencies are smaller. Neubauer and Oleskiewicz [26] model a brake disc using clamped, simply supported and free inner boundary conditions. It is noted in [26] that by tuning the height of the model, for the specific design of brake under investigation, the simply supported inner boundary provides the closest agreement to the measured resonant frequencies over the frequency range of interest.

The closest agreement with the measured resonant frequencies of the disc shown in Table 4 is with the natural frequencies predicted by the spring supported-free model. This result is, perhaps, surprising given the generally applied assumption of a clamped inner boundary at the hat-disc interface $[1,2]$. However, as Chen et al. and Bae and Wickert $[2,15]$ report the bending and in-plane modes of the hatdisc structure change in response to the stiffness and inertia of the hat. Some of this effect may be captured by the springsupported boundary condition.

In Figure 10 the point mobility of the brake disc predicted using the forced vibration model with a spring supported inner boundary is compared to the measured point mobility of the brake disc attached rigidly to a static frame over the frequency range from 1000 to $6400 \mathrm{~Hz}$. For the predicted result, the diametral modes from 1 to 8 were included in the summation. The influence of modes higher than the 8th diametral mode was neglected since the modes are well separated, and, thus, their influence on the point mobility prediction below $6400 \mathrm{~Hz}$ was assumed 
TABLE 4: Comparison of the predicted natural frequencies of the annular plate with different inner boundary conditions with the measured resonant frequencies of the brake disc.

\begin{tabular}{lcccc}
\hline Diametral mode & Clamped, $f_{\text {calc. }}$ & Simply supported, $f_{\text {calc. }}$ & Spring supported, $f_{\text {calc. }}$ & Measured, $f_{\text {meas. }}$ \\
$n$ & $\mathrm{~Hz}$ & $\mathrm{~Hz}$ & $\mathrm{~Hz}$ & $\mathrm{~Hz}$ \\
\hline 2 & 3198 & 1535 & 1325 & 1220 \\
3 & 3784 & 2520 & 2248 & 2196 \\
4 & 4798 & 3895 & 3599 & 3508 \\
5 & 6273 & 5655 & 5367 & 5120 \\
\hline
\end{tabular}

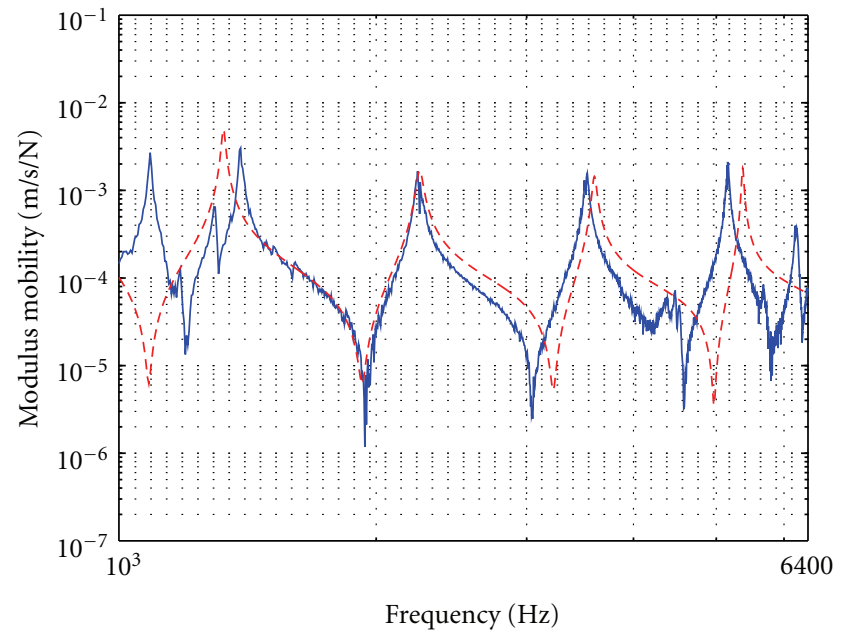

FIGURE 10: Comparison of the modulus of the point mobility of the rigidly mounted brake disc over the frequency range from 1000 to $6400 \mathrm{~Hz}$, logarithmic scale: (dashed line) theoretical prediction for the annular plate with a spring supported inner boundary; (solid line) measured results.

to be marginal. Since the first circumferential $(0,1)$ mode was predicted to occur at $9599 \mathrm{~Hz}$, circumferential modes were not included in the summation. The modal damping values, $\eta_{i}$, for the diametral modes from 2 to 5 were estimated experimentally using the decay rate method. For the remaining diametral modes a damping loss factor was calculated by averaging the measured loss factors of the 2nd to 5th modes. It can be seen in Figure 10 that for the diametral modes of interest, from $n=2$ to $n=5$, the theoretical model indicates relatively good agreement with the measured results. Differences between the predicted natural frequencies and the measured resonant frequencies may be due to the absence of torsional stiffness in the spring supported inner boundary model where only a transverse stiffness was included. The slight differences in peak amplitudes and resonant frequencies between the measured results shown in Figures 9 and 10 may be due to the different levels of torque applied in the hub-mounting procedure between tests.

\section{Summary}

The aim of this paper has been to investigate the effectiveness of two simplified mathematical models that predict the out-of-plane vibrational behaviour of an automotive brake disc. In the first approach the disc vibration is assumed to act in only one dimension. Thus, the disc is modelled as a straight beam in flexure with cyclosymmetric boundary conditions. Predictions of the point mobility made using a wave-based approach and a modal summation approach gave identical results. Comparison of the predicted results with measurements made on both a large diameter ring and a small diameter annular plate formed by removing the central hat of a nonvented brake disc showed good agreement over the frequency range covered by the $n=3$ to $n=5$ diametral modes. Both measured and predicted results follow the trend line of the modulus of point mobility of the equivalent infinite beam structure, indicating beamlike bending wave vibrational behaviour of both structures. Curvature effects appear significant at low frequencies, for example, the frequency range covered by the diametral $n=2$ mode. A calculation of $10 \%$ of the ring frequency can act as useful guide to the lower-frequency limit of the cyclosymmetric straight beam model. As noted by Flint and Hultén $[9,10]$, circumferential modes of the annular ring are not included in the cyclosymmetric beam model. Thus, care should be taken in interpreting the predicted results of the beam model as the lowest circumferential modes of a practical brake disc may occur in the frequency range of interest covered by the $n=2$ to $n=5$ diametral modes.

In the second approach the brake disc was modelled as an annular plate. The inner boundary of the disc at its central hat location was modelled using four different boundary conditions: (i) spring supported, (ii) simply supported, (iii) clamped, and (iv) free. The inclusion of a nonfree boundary condition at the central hat location changed the character of the vibration from beam-like to platelike. Thus, the assumption of a free boundary condition at the central hat location gave least agreement with the measured results from a standard production brake disc. Comparison of the calculated natural frequencies predicted using these models and measurements made upon a standard production nonvented brake disc showed the best agreement with the spring supported boundary condition. The springsupported boundary condition was shown to vary in effect between a free boundary and a simply supported boundary. The clamped boundary condition was the least satisfactory. A comparison of the measured point mobility of a rigidly mounted disc and a prediction of the forced response of the disc made using the spring supported boundary condition showed good agreement over the frequency range covered by the main diametral modes of interest, $n=2$ to $n=5$. 
Both measured and predicted results followed the trend line of the equivalent infinite plate indicating platelike bending wave vibration of the disc.

\section{References}

[1] F. Chen, "Automotive disk brake squeal: an overview," International Journal of Vehicle Design, vol. 51, no. 1-2, pp. 39-72, 2009.

[2] F. Chen, A. T. Tan, and R. L. Quaglia, Disc Brake Squeal: Mechanisms, Analysis, Evaluation, and Reduction/Prevention, SAE International, 2006.

[3] H. Ouyang, W. Nack, Y. Yuan, and F. Chen, "Numerical analysis of automotive disc brake squeal: a review," International Journal of Vehicle Noise and Vibration, vol. 1, pp. 207-231, 2005.

[4] N. M. Kinkaid, O. M. O’Reilly, and P. Papadopoulos, "Automotive disc brake squeal," Journal of Sound and Vibration, vol. 267, no. 1, pp. 105-166, 2003.

[5] A. Papinniemi, J. C. S. Lai, J. Zhao, and L. Loader, "Brake squeal: a literature review," Applied Acoustics, vol. 63, no. 4, pp. 391-400, 2002.

[6] P. Ioannidis, P. C. Brooks, D. C. Barton, and M. Nishiwaki, "Brake system noise and vibration-a review," Braking, pp. 53-73, 2002.

[7] M. Trichês Jr., S. N. Y. Gerges, and R. Jordan, “Analysis of brake squeal noise using the finite element method: a parametric study," Applied Acoustics, vol. 69, no. 2, pp. 147-162, 2008.

[8] Y. Dai and T. C. Lim, "Suppression of brake squeal noise applying finite element brake and pad model enhanced by spectral-based assurance criteria," Applied Acoustics, vol. 69, no. 3, pp. 196-214, 2008.

[9] J. Flint and J. Hultén, "Lining-deformation-induced modal coupling as squeal generator in a distributed parameter disc brake model," Journal of Sound and Vibration, vol. 254, no. 1, pp. 1-21, 2002.

[10] J. Flint, "Modelling of high-frequency disc-brake squeal," Brakes, pp. 39-50, 2000.

[11] H. Ouyang, J. E. Mottershead, M. P. Cartmell, and M. I. Friswell, "Friction-induced parametric resonances in discs: effect of a negative friction-velocity relationship," Journal of Sound and Vibration, vol. 209, no. 2, pp. 251-264, 1998.

[12] H. Ouyang and J. E. Mottershead, "Unstable travelling waves in the friction-induced vibration of discs," Journal of Sound and Vibration, vol. 248, no. 4, pp. 768-779, 2001.

[13] H. Ouyang, Q. Cao, J. E. Mottershead, and T. Treyde, "Vibration and squeal of a disc brake: modelling and experimental results," Proceedings of the Institution of Mechanical Engineers, Part D: Journal of Automobile Engineering, vol. 217, no. 10, pp. 867-875, 2003.

[14] Q. Cao, H. Ouyang, M. I. Friswell, and J. E. Mottershead, "Linear eigenvalue analysis of the disc-brake squeal problem," International Journal for Numerical Methods in Engineering, vol. 61, no. 9, pp. 1546-1563, 2004.

[15] J. C. Bae and J. A. Wickert, "Free vibration of coupled disk-hat structures," Journal of Sound and Vibration, vol. 235, no. 1, pp. $117-132,2000$.

[16] S. Bashmal, R. Bhat, and S. Rakheja, "Frequency equations for the in-plane vibration of circular annular disks," Advances in Acoustics and Vibration, vol. 2010, Article ID 501902, 8 pages, 2010.

[17] D. Boennen, Analytical beam and annular plate models for automotive disc brake vibration [Ph.D. thesis], Loughborough University, 2008.

[18] D. Boennen and S. J. Walsh, "Investigation of analytical beam and annular plate models for automotive disc brake vibration," Braking, pp. 264-274, 2006.

[19] L. Cremer, M. Heckl, and E. E. Ungar, Structure-Borne Sound: Structural Vibrations and Sound Radiation at Audio Frequencies, Springer, 1988.

[20] S. M. Vogel and D. W. Skinner, "Natural frequencies of transversely vibrating uniform annular plates," Journal of Applied Mechanics, vol. 32, pp. 926-931, 1965.

[21] A. W. Leissa, Vibration of Plates, NASA SP-160, Ohio State University, 1969.

[22] R. V. Southwell, "On the free vibration of a uniform circular disc clamped at its centre; and on the effects of rotation," Proceedings of the Royal Society, vol. 101, pp. 133-153, 1928.

[23] C. T. Wang, Applied Elasticity, McGraw-Hill, 1953.

[24] S. J. Walsh and R. G. White, "Mobility of a semi-infinite beam with constant curvature," Journal of Sound and Vibration, vol. 221, no. 5, pp. 887-902, 1999.

[25] D. Thompson, Railway Noise and Vibration: Mechanisms, Modelling and Means of Control, Elsevier Science, 2008.

[26] M. Neubauer and R. Oleskiewicz, "Brake squeal control with shunted piezoceramics-efficient modelling and experiments," Proceedings of the Institution of Mechanical Engineers, Part D: Journal of Automobile Engineering, vol. 222, no. 7, pp. 1141-1151, 2008. 

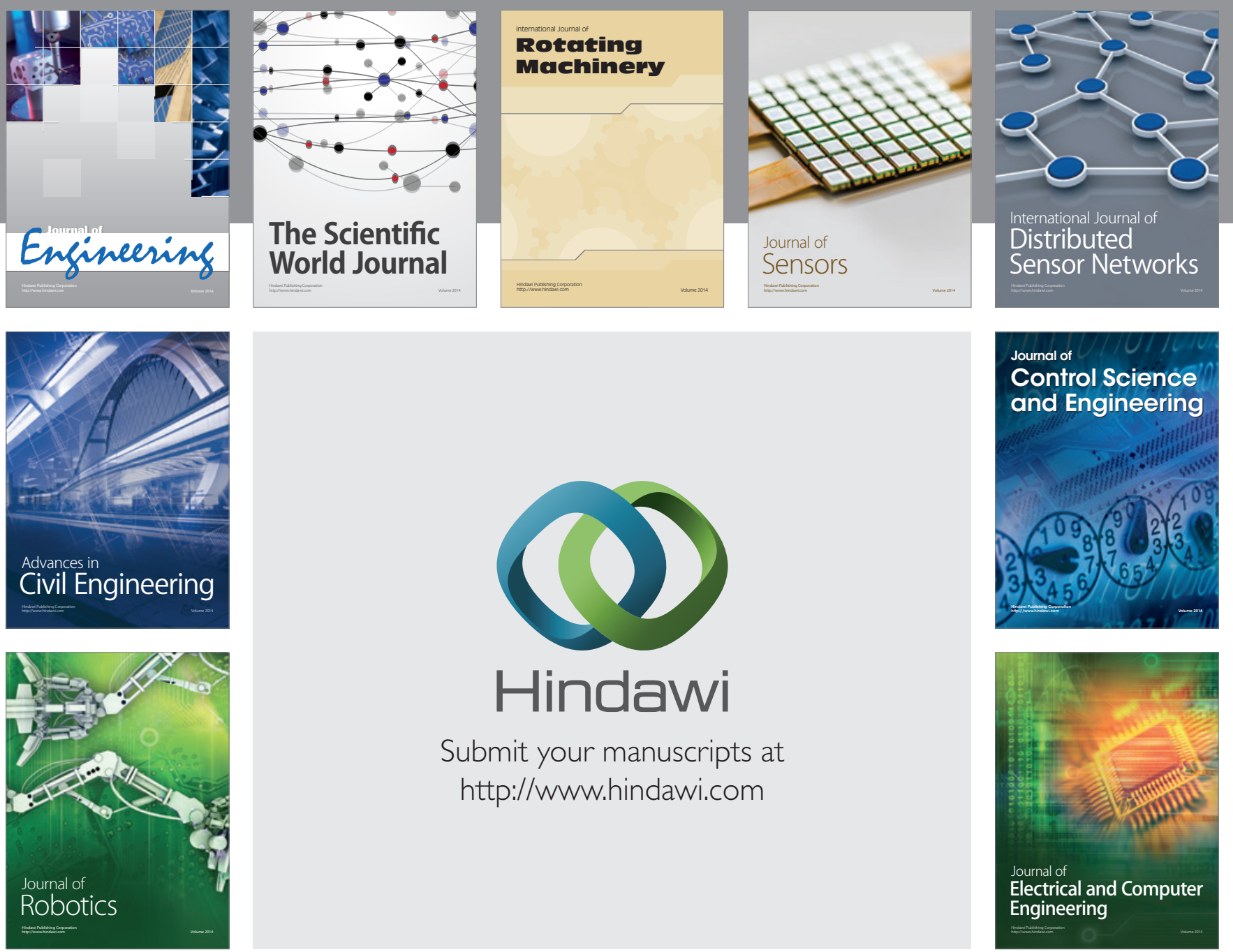

Submit your manuscripts at

http://www.hindawi.com
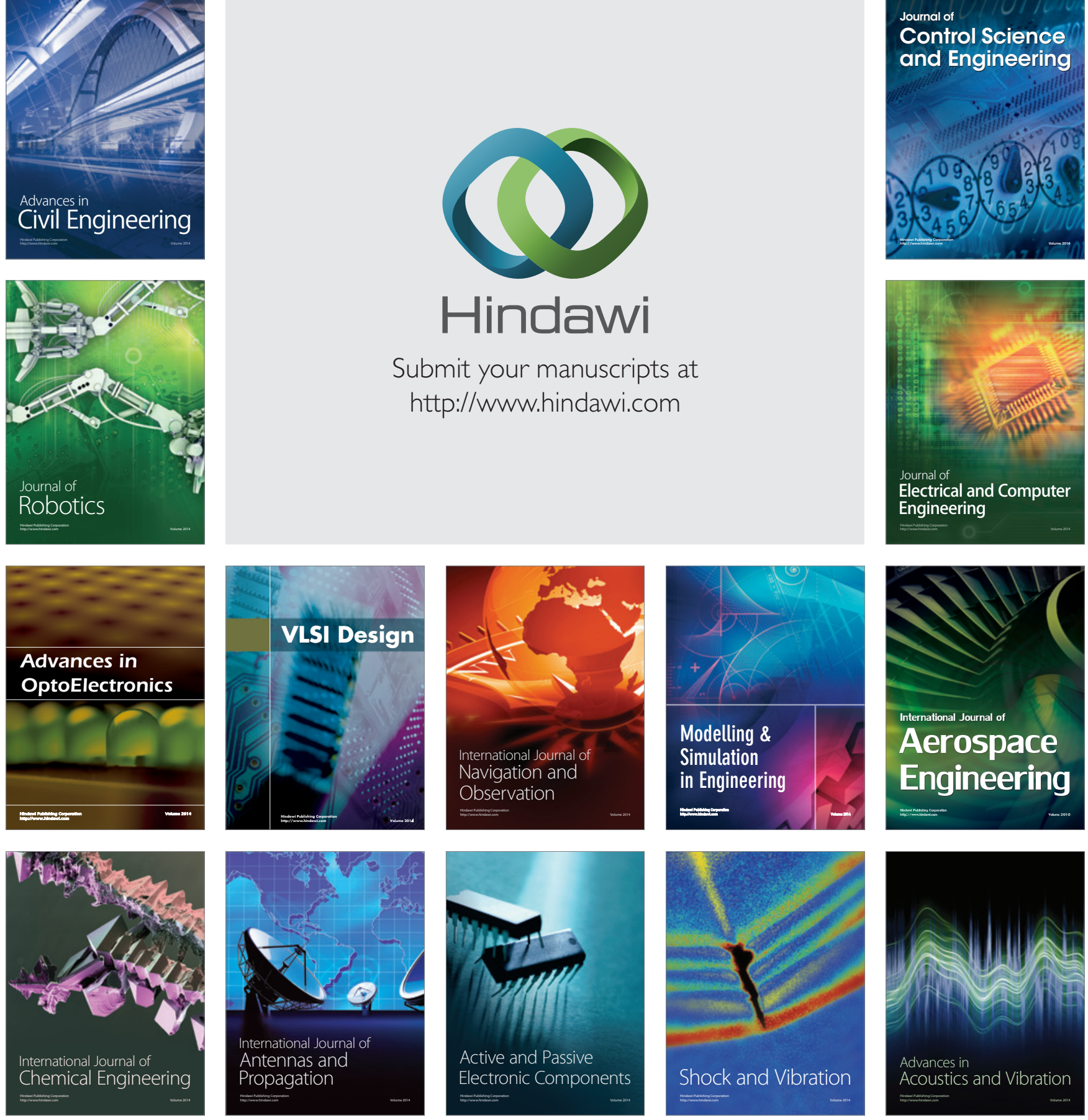